\title{
Project Management of Randomized Clinical Trials: A Narrative Review
}

\author{
Hamidreza Goodarzynejad ${ }^{1, *}$; Abdolreza Babamahmoodi $^{2}$ \\ ${ }^{1}$ Department of Cardiac Research, Tehran Heart Center, Tehran University of Medical Sciences, Tehran, IR Iran \\ ${ }^{2}$ Health Management Research Center, Baqiyatallah University of Medical Sciences, Tehran, IR Iran \\ ${ }^{*}$ Corresponding Author: Hamidreza Goodarzynejad, Department of Cardiac Research, Tehran Heart Center, Tehran University of Medical Sciences, Tehran, IR Iran. Tel: +98-2188029245, \\ Fax: +98-2188029256, E-mail: hami_nag@yahoo.com; h-goodarzynejad@razi.tums.ac.ir
}

Received: April 16, 2013; Revised: June 2, 2015; Accepted: June 10, 2015

\begin{abstract}
Context: A well-structured protocol for a clinical trial may be able to answer clinical questions, but it cannot be deemed enough to ensure success in the face of incompetent management of time as well as human and economic resources. To address this problem, in this article, we present our literature review on evidence as to how a good knowledge of proper management among researchers can enhance the likelihood of the success of clinical trial projects.

Evidence Acquisition: Using multiple search strategies, we conducted a literature review on published studies in the English language from 2002 to 2012 by searching the Cochrane Database of Systematic Reviews, MEDLINE, Google Scholar, and EMBASE.

Results: Our review suggests that a successful trial requires a work plan or work scope as well as a timeline. The trial manager should subsequently manage the study in accordance with the plan and the timeline. Many research units have called for a clinical project manager with scientific background and regulatory skills to effect coordination among various aspects of a clinical trial.

Conclusions: Project management may benefit both the managerial and scientific aspects of medical projects and reduce fund waste. However, little has been written to date on project management in the context of clinical research. The suggestions represent the views of the individual authors. To provide a high level of evidence in this regard, we recommend that a randomized controlled trial be performed to compare trial projects progressed with and without the use of project management.
\end{abstract}

Keywords: Clinical Trial; Project Management; Trial Manager; Principal Investigator; Study Protocol

\section{Context}

Large randomized controlled trials (RCTs) are frequently considered the reference standard for producing high levels of scientific evidence to guide clinical practice $(1,2)$. In an attempt to overcome inconsistencies throughout the world, the guideline for good clinical practice (GCP) was approved on 17 July 1996 and implemented for clinical trials from 17 January 1997 (3, 4). The GCP was followed by a large number of decrees and circulars, increasing the regulatory complexity for the authorization and conduct of clinical trials. The increase in regulatory issues went hand in hand with the refinement of methodologies and research techniques. The inclusion of fewer patients than is required usually results in extended trial periods and raised costs. Hence, one of the most obvious challenges in a trial is to optimize the recruitment of the required number of patients in a timely and efficient manner (5).

Excellent leadership and negotiation and communication skills optimize managing project deliverables, patient recruitment practices, and compliance with regulatory requirements. On the other hand, a poorly managed clinical trial can lead to product approval delays and increased costs. Moreover, it must not be forgotten that the development of a new drug from synthesis to marketing takes on average 12 years and costs more than 250 million USD (6), and of more than 10000 molecules synthesized only 1 or 2 will hit the market (7). If it is true that a wellstructured protocol can answer clinical questions, it is equally true that this is not enough to ensure the success of a study at risk of failure due to inept management (8).

In the last decades, although a systematic approach to project management has been applied in engineering and construction industries, there is a dearth of data on project management in the context of clinical research. The purpose of this review is to provide an overview of the published literature on how the knowledge of project management can increase the chance of the success of clinical trial projects.

\section{Evidence Acquisition}

We searched published studies in the English language from 2002 to 2012 in the Cochrane Database of Systematic Reviews, MEDLINE, Google Scholar, and EMBASE. We reviewed the titles of articles for relevant content, discarded unrelated articles, subjected the remaining articles to abstract review, and subsequently reviewed the selected relevant papers in detail. Finally, we hand searched the reference lists of key studies and reviews and citations thereof to identify any additional articles not captured by the main search strategy.

Copyright ( 2015, Iranian Red Crescent Medical Journal. This is an open-access article distributed under the terms of the Creative Commons Attribution-NonCommercial 4.0 International License (http://creativecommons.org/licenses/by-nc/4.0/) which permits copy and redistribute the material just in noncommercial usages, provided the original work is properly cited. 


\section{Results}

A clinical trial has many characteristics in common with other types of business projects as is defined in the project management domain (8). These characteristics include but not limited to the following:

- There are pre-defined objectives to be achieved.

- Identified resources are necessary to achieve these objectives.

- Activities should be planned to achieve the objectives.

- The work should be monitored constantly.

- The criteria for evaluating the results should be defined.

- The results should be evaluated.

- The project is closed when the objectives have been achieved or when the objectives will not or cannot be met, or when the need for the project no longer exists.

\subsection{Clinical Trials Project Management}

Clinical trial management is most simply defined as the process that an organization follows to ensure that quality (defined as minimized risks and clean data) is delivered efficiently and punctually (9). It refers to a standards-driven process that a project manager initiates and follows in order to successfully manage clinical trial sites, clinical research associates, and workflow by using clinical trial management tools or software (9). Prolonged timelines and heavy costs related to large trials have prompted a new focus on more efficient clinical trial management (10). It is possible to dramatically reduce the total cost of a clinical trial by $60 \%$ - $90 \%$ without compromising the scientific validity of the results (11). Given the modest effect of the most beneficial treatments and the dangers inherent in failing to identify risks before the marketing of cardiovascular drugs, in particular, the importance of clinical trial expenses is increasing in cardiovascular medicine, where the emphasis is on large trials with adequate sample sizes in order to detect the clinically relevant differences (12). The reduction in the sample size would decrease expenses, but it is not likely to provide adequate power to determine the risks and benefits of a new drug.

\subsection{The Clinical Trial Project Plans}

Similar to many projects in general that are not completed on time, on budget (13-15), and to the expected quality (16), many clinical trials fail to deliver due to the absence of a structured, practical, businesslike approach to trial management. There is no clearly defined code for the management practice of a clinical trial (16). Although many successful RCTs have been previously produced and have changed clinical practice, the knowledge and expertise achieved on how to deliver such trials have not been broadly published (16). Lengthy approaches for reviewing the literature, developing a protocol, attracting the fund, and designing data collection forms usually exist; however, rarely does the same approach exist for the management of clinical trial projects (17).
Each trial requires the development of a work plan (or scope-of-work document) that can be used to clearly define which department is responsible for which task when the study is being conducted by multiple departments out of a single institution. Likewise, the same document can be drawn upon to define responsibilities if multiple institutions (e.g., a coordinating center and a statistical center) are involved. The more the detail included in this document in advance, the more likely it is that the important tasks will not be missed and that each group is not assuming that someone else is responsible for the task. A scope-of-work document can also be utilized to help with budgeting for the different members of the team.

Another critical document to create as part of the initial planning requirements is a timeline with realistic milestones. There are many tools that can be exploited to create a timeline, including Microsoft Project software. Appropriate Gantt charts and resource planning are part of Microsoft Project, but unfortunately not all trial managers either have this readily available resource or know how to input the needed data to make it a useful tool. The timeline, in conjunction with the scope-of-work document, will collectively provide the roadmap for the overall project.

\subsection{The Life Cycle of a Clinical Trial}

A more accurate control, regardless of the therapeutic area or trial stages (all the way through from 'preclinical' phase 1 to 'post-approval' phase 4 studies), is ensured by typically breaking down the life cycle of each clinical trial project into 4 phases: conceptual, planning, implementation, and analysis (Figure 1). A project is based on input, which is normally supplied by the sponsor of the project and generates the output (Figure 2). An input is the objective to be achieved in a specific predetermined time (enrollment of X evaluable subjects in X months, the sponsor inclusion in the database of data collected in X days, etc.) and is generally described in the contract. A clinical study produces an output that will ultimately help provide answers to the study objectives; these results are described in a clinical study report that states formally the end of the study. As we move through the implementation phase, the amount of work and number of players involved increase significantly and this is where project management becomes more critical. Monitoring is an essential element for project management and is constantly done throughout the duration of the project. It allows managers to collect measures and disseminate performance information in order to identify areas that need special attention and to implement any action improvement (18). A phase of a project usually ends with a review of the tasks and monitoring of the objectives (Figure 1).

\subsection{The Clinical Trial Protocols}

A protocol is a document that describes the purpose, design, methodology, statistical considerations, and organization of a study and provides basic information and 
rationale for the clinical study. The contents that should be present in the protocol are described by the GCP. The protocol writing is a task for one person, usually the principal investigator, not a committee (19). The principal investigator should also be supportive and committed, and be available to take the lead on clinical or scientific issues. However, it is not necessary for this person to be involved in the dayto-day running of the trial. It is the responsibility of a trial manager to check how the protocol will be put into practice on a day-to-day basis (19). Although a well-structured protocol can answer scientific methodological questions during the study, projects may fail even with well-written protocols if it is not meticulously considered how the protocol will be put into practice on a day-to-day basis. The principal investigator and the trial manager, therefore, have overall responsibility for delivering the trial.

\subsection{Roles and Key Characteristics of a Trial Manager}

The project manager is the person responsible for managing the project in order to achieve the objectives in respect of cost and time as well as the technical quality of the result. While trial managers are not required to complete all the tasks, they are responsible for ensuring that all aspects of the project are planned, implemented, monitored, and controlled on a daily basis, and project outputs and outcomes are obtained (20). Unfortunately, the responsibilities of this role are complex and the body of knowledge available to guide trial managers is still very limited. As Farrell et al. (8) suggested: "It is time to produce standard trial management guidelines and develop robust methods of evaluation." In a research project, "Alcohol and Pregnancy Project", (21) conducted in Australia, at the completion of the project, the researchers were asked via a questionnaire to express their opinion about the application of project management for medical research projects. The responders agreed that project management contributes substantially to the research and emphasized that trial manager characteristics are of essential importance within the team.

A perfect project plan will not ensure the success of a project without its implantation by a strong clinical trial coordinator or manager. The requirements of a clinical project manager are similar to those of any other project manager (communication and presentation skills, ability to organize and motivate others, enthusiasm, drive innovation and leadership, ability to manage the budget of the study and monitor costs, strategic and operational skills to plan and conduct a study, capacity to mediate between the various figures), but also add to these scientific competence and motivation (8). The manager can detect and correct errors that are sometimes severe (e.g., protocol deviations, deviations in consent), emphasizing the importance of monitoring throughout the conduct of the study (22). Regular and constant complete communication between the trial manager with the principal investigator and other stakeholders is essential to make sure all aspects remain on track as per the original plan. The figures of a good clinical trial coordinator may be summarized as 30\% research knowledge and technical skills and 70\% communication skills (9).

Figure 1. A Typical Life Cycle of a Clinical Trial Project With its Phases and Activities

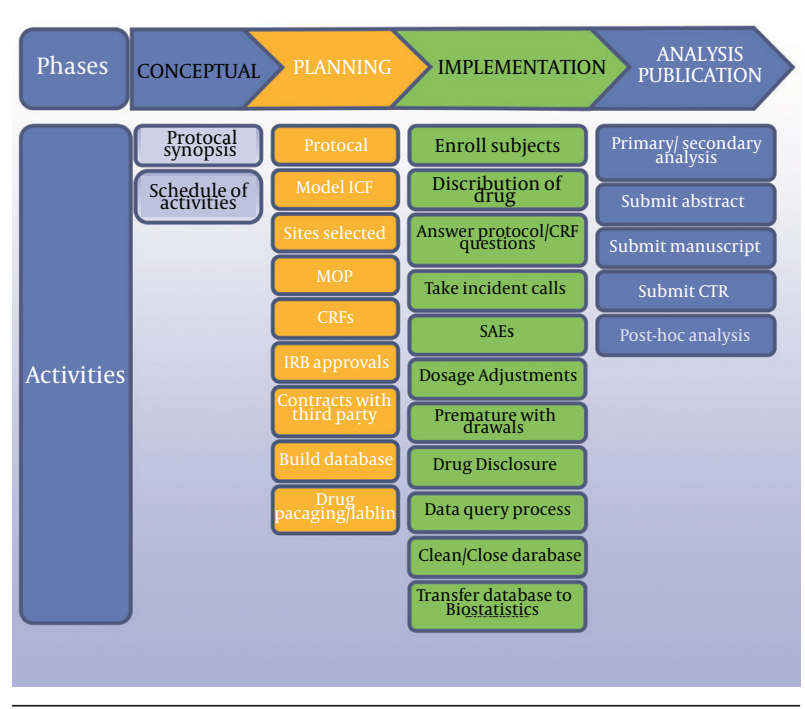

ICF, informed consent form; MOP, manual of procedures; CRF, case report form; IRB, institutional review board; SAE, serious adverse event; and CTR, clinical technical report.

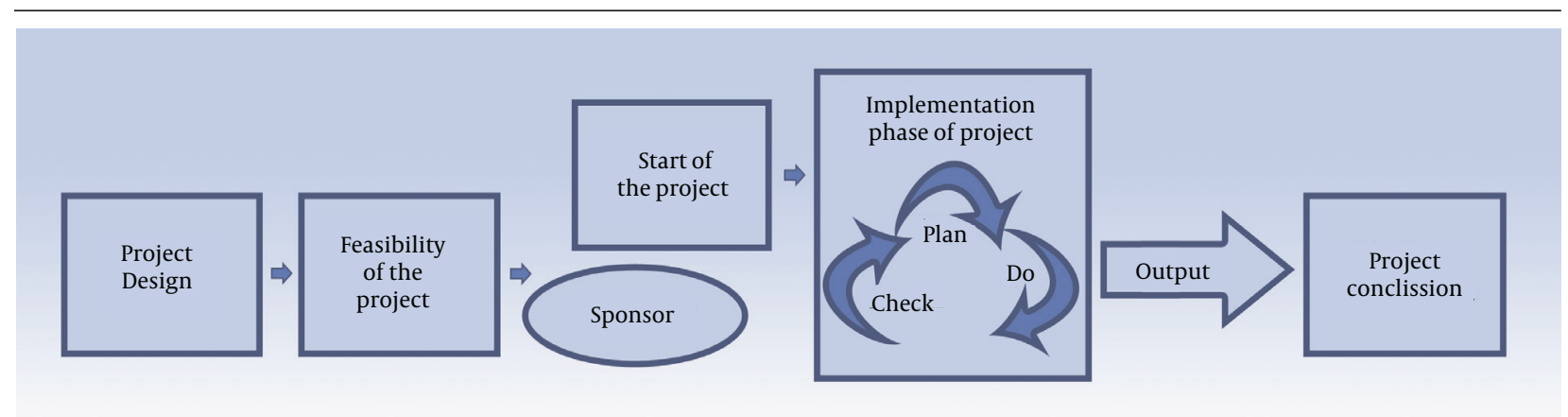

Figure 2. The Phases of a Typical Project 


\subsection{Challenges of Project Management in Clinical Trials}

Clinical trials all need the same coordinated processes and systems, irrespective of the size, scope, costs, or period. The key challenge is then to implement and maintain effective management systems and techniques in response to the needs of the trial project (23). Drug and biologics development projects have to contend with several factors, including inability to control patient registration, unpredictability of clinical outcomes (e.g., adverse events), and reliance on regulatory agencies (e.g., the FDA in the United States), for the approval of a new drug, which influences schedule and budget as the key performance indicators of project management. As a result, even with the application of project management, the best we can do is to improve our predictions for the launch dates of new products into market.

Another apparent challenge in clinical trials is to register the patient sample size as timely and efficiently as possible (5). Each clinical trial is unique because one tiny difference in the protocol needs between two trials may lead to drastic changes in yielding recruitment lifecycle and register timing parameters. Many factors such as inclusion/exclusion criteria, visit schedule, recruitment tactics, placebo allotment, development phase, and patient motivation can potentially affect the final number of patients who enroll in the trial. A lower number of available patients than is expected usually results in an extended length of time to recruit a certain number of patients and increased costs. Multicenter trials provide highyielding recruitment strategies that can be advantageous if a large number of patients is required and also offer the advantage of higher generalizability of the findings $(24,25)$. However, performing international multicenter trials requires a complex organization, a dedicated and experienced methods center, and motivated participating investigators across the different sites $(8,26)$. In the strategies for trial enrollment and participation study (STEPS) (27), an analysis of 114 multicenter trials demonstrated that $45 \%$ failed to reach $80 \%$ of the pre-specified sample size. Less than one-third enrolled their original target number of participants within the time originally specified, and around one-third had extensions in time and resources. Among the factors observed more often in trials that recruited successfully was having a dedicated trial manager (odds ratio: $3.80,95 \% \mathrm{CI}$ : 0.79 to $36.14 ; \mathrm{P}=$ 0.087) (27).

Identifying appropriate clinical sites and setting up realistic enrollment expectations are also challenges in multicenter studies. Because site principal investigators often lack the time to spend on administrative tasks, it is crucial to have a trial coordinator who could adopt much of the time. When making a decision to participate, sites find it more important to have sufficient support than to be offered a financial compensation for participation (28, 29). In addition, the presence of a trial manager will fa- cilitate an appropriate study infrastructure for the daily management of the study (30).

Finally, achieving ethics approval is often a lengthy process because procedures, documents, and legislation may vary among participating sites in different countries (31). It is, therefore, recommended to have dedicated and well-trained trial staff to accomplish these administrative tasks (32-34).

The STEPS collaborators (27) proposed that the dimensions of conducting a successful trial comprise marketing, sales, and ongoing client management and identified that in the recruitment stage of a trial, the most demanding activity is to establish and implement a range of effective management techniques parallel to those used in a successful business. They also suggested that future trials consider the different needs at different phases in the life of trials and focus on the process of actually performing trials (27).

\subsection{Central Management Versus Local Manage- ment}

A complete patient follow-up and high-quality data collection in a large multicenter or multinational trial require the establishment of a relatively complex organization (35). Individual sites generally arrange their local ethics procedures and trial logistics and receive per-patient payments as compensation. Alternatively, a single trial manager may handle all trial-related tasks for multiple sites in a certain geographic area. The fixation using alternative implants for the treatment of hip fractures (FAITH) trial (36) aimed to assess how these two different strategies affect the performance of the trial. In this study, trial progression in the Netherlands, where a central trial manager performed most tasks for multiple centers in a defined geographical area, was better than that in Canada and the United States, where local research assistants were appointed at individual sites. Although the collection of follow-up data was equally adequate in both systems, the central trial management system was related to a shorter trial start-up time, higher inclusion rate, and higher inclusion percentage (36). Furthermore, the availability of the central trial manager in the Netherlands made it possible for smaller community hospitals to participate in the FAITH multicenter trial. These sites generally lack infrastructure for proper study conduct or the local site's coordinator is normally unable or reluctant to participate in such trials. Be that as it may, these centers treat more patients from the targeted population (e.g., femoral neck fracture patients in the FAITH trial) than large university hospitals. Thus, the availability of a central trial manager can positively influence the recruitment rate.

\section{Conclusions}

The numerous and complex regulatory issues introduced into the management of clinical trials according 
to the GCP as well as medical science advances in methodologies and procedures have rendered the field of clinical research extremely complex. This complexity, however, has conferred a tremendous benefit for both parties involved in the studies: For patients by providing a guarantee for their safety and respecting their rights and for investigators by providing guidelines that, if carefully followed, guarantee valid results. In addition, investigators, who are employed to implement the GCP in the conduct of clinical studies, have also begun to apply them to clinical practice with clear benefits for patients. This has significantly increased the workload of investigators and the need for having a project management with scientific background, knowledge, and regulatory skills in conducting clinical research. The clinical project manager is a professional who applies the definition of project management to the field of clinical research to ensure that all stages of a clinical trial are properly managed, that the objectives of the trial are achieved on time, on budget, and according to the GCP, and that the safety of the subjects participating in the clinical trial and the quality of collected data are guaranteed.

There are many challenges associated with the management of clinical trial projects. One of the most obvious challenges in trials is to register an efficient sample size of patients in a timely fashion. Another challenge in a multicenter study is to identify appropriate clinical sites and set up realistic recruitment expectations. Finally, the key challenge is to implement and maintain effective management systems and techniques in response to the needs of the trial project.

Project management in health and medical research may substantiallybenefit both the managerial and scientific aspects of medical projects. It is also suggested that project management could reduce a proportion of fund waste. However, there is still no randomized controlled trial to compare trial projects progressed with and without the use of project management. We recommend such a comparison to provide a high level of evidence in this regard.

\section{Acknowledgements}

We would like to thank Ms. Zahra Aghazadeh for her assistance with the preparation of the images.

\section{Authors' Contributions}

Hamidreza Goodarzynejad was involved in drafting the manuscript. Abdolreza Babamahmoodi revised the manuscript and gave final approval of the version to be published. All authors read and approved the final manuscript.

\section{References}

1. McLeod RS. Issues in surgical randomized controlled trials. World J Surg. 1999;23(12):1210-4.

2. Yusuf S, Collins R, Peto R. Why do we need some large, simple randomized trials? Stat Med.1984;3(4):409-22.
3. Otte A, Maier-Lenz H, Dierckx RA. Good clinical practice: historical background and key aspects. Nucl Med Commun. 2005;26(7):563-74.

4. Guideline ICH Harmonised Tripartite. E6: Note for Guidance on Good Clinical Practice. London: PMP/ICH/135/95). European Medicines Agency; 2002.

5. Thoma A, Farrokhyar F, McKnight L, Bhandari M. Practical tips for surgical research: how to optimize patient recruitment. Can J Surg. 2010;53(3):205-10.

6. Heilman RD. Drug development history, "overview," and what are GCPs? Qual Assur. 1995;4(1):75-9.

7. Baldi A. Computational approaches for drug design and discovery: An overview. Sys Rev Pharm. 2010;1(1):99-105.

8. Farrell B, Kenyon S, Shakur H. Managing clinical trials. Trials. 2010;11:78.

9. Richardson B, Newman RW. Clinical Trial Management -An Integrated Process. Drug Development; 2006.

10. Bose A, Das S. Trial analytics--a tool for clinical trial management. Acta Pol Pharm. 2012;69(3):523-33.

11. Eisenstein EL, Collins R, Cracknell BS, Podesta O, Reid ED, Sandercock $\mathrm{P}$, et al. Sensible approaches for reducing clinical trial costs. Clin Trials. 2008;5(1):75-84.

12. Eisenstein EL, Lemons P2, Tardiff BE, Schulman KA, Jolly MK, Califf RM. Reducing the costs of phase III cardiovascular clinical trials. Am Heart J. 2005;149(3):482-8.

13. Howe R, Flanagan C. Case managers getting it done: a project management primer. Lippincotts Case Manag. 2004;9(3):152-4.

14. Lyneis JM, Cooper KG, Els SA. Strategic management of complex projects: a case study using system dynamics. Syst Dyn Rev. 2001;17(3):237-60.

15. Nevan Wright J. Time and budget: the twin imperatives of a project sponsor. Int J Proj Manag. 1997;15(3):181-6.

16. Gardiner PD, Stewart K. Revisiting the golden triangle of cost, time and quality: the role of NPV in project control, success and failure. Int J Proj Manag. 2000;18(4):251-6.

17. Farrell B. Efficient management of randomised controlled trials: nature or nurture. BMJ.1998;317(7167):1236-9.

18. Freeman JV, Cole TJ, Chinn S, Jones PR, White EM, Preece MA. Cross sectional stature and weight reference curves for the UK, 1990. Arch Dis Child.1995;73(1):17-24.

19. Warlow C. How to do it. Organise a multicentre trial. Bmj. 1990;300(6718):180-3.

20. Martin P, Tate K. Getting Started in Project Management. Toronto: John Wiley \& Sons, Inc; 2001.

21. Payne JM, France KE, Henley N, D'Antoine HA, Bartu AE, Elliott EJ, et al. Researchers' experience with project management in health and medical research: results from a post-project review. BMC Public Health. 2011;11:424.

22. Rai K. Integrated monitoring: Setting new standards for the next decade of clinical trial practice. Perspect Clin Res. 2011;2(1):28-33.

23. Francis D, Roberts I, Elbourne DR, Shakur H, Knight RC, Garcia J, et al. Marketing and clinical trials: a case study. Trials. 2007;8:37.

24. Bossert EA, Evans S, Van Cleve L, Savedra MC. Multisite research: a systems approach. J Pediatr Nurs. 2002;17(1):38-48.

25. Bhandari M, Schemitsch EH. Beyond the Basics. Tech Orthop. 2004;19(2):83-7.

26. Sprague S, Matta JM, Bhandari M, Anterior Total Hip Arthroplasty Collaborative I, Dodgin D, Clark CR, et al. Multicenter collaboration in observational research: improving generalizability and efficiency. J Bone Joint Surg Am. 2009;91 Suppl 3:80-6.

27. Campbell MK, Snowdon C, Francis D, Elbourne D, McDonald AM, Knight R, et al. Recruitment to randomised trials: strategies for trial enrollment and participation study. The STEPS study. Health Technol Assess. 2007;11(48):iii.

28. Zurbuchen U, Schwenk W, Bussar-Maatz R, Wichlas F, Buhr HJ, Neudecker J, et al. [Clinical studies outside university clinics : What are the problems to implement this?]. Chirurg. 2010;81(2):160.

29. Raftery J, Kerr C, Hawker S, Powell J. Paying clinicians to join clinical trials: a review of guidelines and interview study of trialists. Trials. 2009;10:15. 
30. Irving SY, Curley MA. Challenges to conducting multicenter clinical research: ten points to consider. AACN Adv Crit Care. 2008;19(2):164-9.

31. Van Lieshout EM, Zielinski SM. Surviving a site audit: tips for good clinical practice in an implant trial. J Long Term Eff Med Implants. 2009;19(3):215-22.

32. Kidd TJ, Marks GB, Bye PT, Wainwright CE, Robinson PJ, Rose BR, et al. Multi-centre research in Australia: analysis of a recent $\mathrm{Na}-$ tional Health and Medical Research Council-funded project. Respirology. 2009;14(7):1051-5.

33. van Santvoort HC, Besselink MG, Gooszen HG. [Obtaining medical ethical approval for a multicentre, randomised study: prospective evaluation of a ponderous process]. Ned Tijdschr Ge- neeskd. 2008;152(38):2077-83.

34. Sherwood ML, Buchinsky FJ, Quigley MR, Donfack J, Choi SS, Conley SF, et al. Unique challenges of obtaining regulatory approval for a multicenter protocol to study the genetics of RRP and suggested remedies. Otolaryngol Head Neck Surg. 2006;135(2):189-96.

35. Sprague S, Leece P, Bhandari M, Tornetta P3, Schemitsch E, Swiontkowski MF, et al. Limiting loss to follow-up in a multicenter randomized trial in orthopedic surgery. Control Clin Trials. 2003;24(6):719-25.

36. Zielinski SM, Viveiros H, Heetveld MJ, Swiontkowski MF, Bhandari M, Patka P, et al. Central coordination as an alternative for local coordination in a multicenter randomized controlled trial: the FAITH trial experience. Trials. 2012;13:5. 